\title{
Interplay between stick-slip motion and structural phase transitions in dry sliding friction
}

\author{
A. Buldum and S. Ciraci \\ Department of Physics, Bilkent University, Bilkent 06533, Ankara, Turkey \\ (Received 15 January 1997; revised manuscript received 5 February 1997)
}

\begin{abstract}
Simulations of dry sliding friction between a metal asperity and an incommensurate metal surface reveal unusual atomic processes. The lateral force exhibits a quasiperiodic variation with the displacement of an asperity; each period consists of two different stick-slip processes involving structural transitions. While one layer of asperity changes and matches the substrate lattice in the first slip, two asperity layers merge into a new one through a structural transition during the second slip. This leads to wear. The lateral force decreases abruptly during these slip stages, but it increases between two consecutive slips and resists the relative motion. The analysis of the order suggests that each structural transition is associated with a first-order phase transition. Nonadiabatic atomic rearrangements during these phase transitions involve a new kind of mechanism of energy dissipation in the dry sliding friction. [S0163-1829(97)03720-X]
\end{abstract}

Friction is a resistance to the relative motion of two sliding objects imposed by induced nonconservative forces. This phenomenon is relevant for various fields in science and technology ranging from quantum friction and energy dissipation to wear and tribology, even earthquake science. ${ }^{1}$ While the Amonton's Law (i.e., for a given pair of objects the friction force is proportional to the normal force, $F_{f}=\mu F_{N}$ ) has been commonly used, microscopic features of friction have been investigated in several recent studies. ${ }^{2-5}$ The invention of the atomic force microscope ${ }^{6}$ and the development of friction force microscope (FFM) therefrom ${ }^{7}$ have made a great impact on friction by providing atomic scale investigations. Together with these new developments, the quantum behavior of many-body interactions have been implemented in the friction ${ }^{8-10}$ and extensive simulations of atomic processes taking place in the course of sliding have been provided. ${ }^{3-5,11}$

The dry sliding friction of two objects, that are in direct contact through their asperities, involves many interesting and complex physical phenomena, such as adhesion, wetting, atom exchange, elastic and plastic deformation, and various energy damping processes. The relative motion of two commensurate surfaces can take place through the repeating stick-slip stages. The stick-slip behavior and energy damping therefrom can be easily visualized for the commensurate and weakly deformable sliding surfaces. ${ }^{7,9}$ However, the situation is rather complex if the sliding surfaces are incommensurate and undergo an elastic even plastic deformation involving the atom exchange and wear. In fact, the FFM study for the dry sliding friction between the Ir tip and the $\mathrm{Au}(111)$ surface revealed unusual results. ${ }^{12}$ For example, a distinct stick-slip behavior was absent, and friction was stronger during unloading than during loading with identical values of $F_{N}$. Theoretical works ${ }^{4,11}$ on the dry sliding between metal surfaces have focused on the junction growth ${ }^{13}$ and on the occurance of stick-slip process. The important effects of deformation, such as the reversal of corrugation and second state in the stick-slip motion, have been predicted by calculations based on model systems. ${ }^{14}$

In this paper, we report atomic processes during the contact and subsequent dry sliding friction between a single asperity and flat metal surface. We predict a new stick-slip behavior and energy dissipation processes that are relevant for the sliding between incommensurate metal surfaces where the interfacial contact is not continuous but consists of numerous asperity contacts. Already with the onset of contact, atoms from the lower planes of the asperity become matched to the substrate lattice structure. While the relative motion occurs between the regular plane and the substrate matched plane of the asperity, the strain energy is stored at the interface. Part of the stored energy is then released through the alternating and periodically repeating structural transitions which, in turn, induce an abrupt decrease of the lateral force, as well as wear leading to junction growth.

Our findings are obtained from the state of the art atomic simulations based on the molecular dynamics calculations at $T=4 \quad \mathrm{~K}$ using the realistic embedded atom model potentials. ${ }^{15}$ The asperity is modeled by a pyramid consisting of ten $\mathrm{Ni}(111)$ atomic planes. The metal substrate itself is represented by a $\mathrm{Cu}$ slab consisting of twelve (110) atomic planes. The Ni(111) planes are parallel but incommensurate to the $\mathrm{Cu}(110)$ planes. The motion of the Ni pyramid (asperity) is controlled by changing the lateral and perpendicular position of its top two layers. The sliding occurs in the $x y$ plane along the $x$ direction under a constant loading force $F_{N}=1.65 \mathrm{eV} / \AA$ distributed on the top plane of the Ni asperity. One side of the $\mathrm{Ni}(111)$ equilateral triangle and the shorter side of the $\mathrm{Cu}(110)$ rectangular unit cell are taken perpendicular to the $x$ direction. It should be noted that the atomic scale simulation of sliding with steady-state configuration is rather difficult under a constant loading force $F_{N}$ and constant temperature, and requires special caution. The sliding is provided first by displacing the $x y$ position of atoms in the top two layers by an increment of $0.05 \AA$ in the $x$ direction, and then relaxing the rest of the atoms for 500 steps, each step lasting $10^{-15}$ seconds. The temperature is kept fixed at $4 \mathrm{~K}$ by rescaling the velocities of the all dynamic atoms both in the substrate and asperity. ${ }^{16}$ By increasing the number of relaxation steps from 500 to 3000 we tested that the convergence to steady state is achieved.

Initially, the $\mathrm{Ni}$ asperity under $F_{N}$ is lowered towards the $\mathrm{Cu}$ surface from a separation greater than the equilibrium adsorption distance of Ni. The energy of the whole system is lowered by $\sim 22 \mathrm{eV}$ owing to mainly the adhesive interaction upon formation of the contact. Once the contact is established, the layer structure of the pyramid remains, but single 


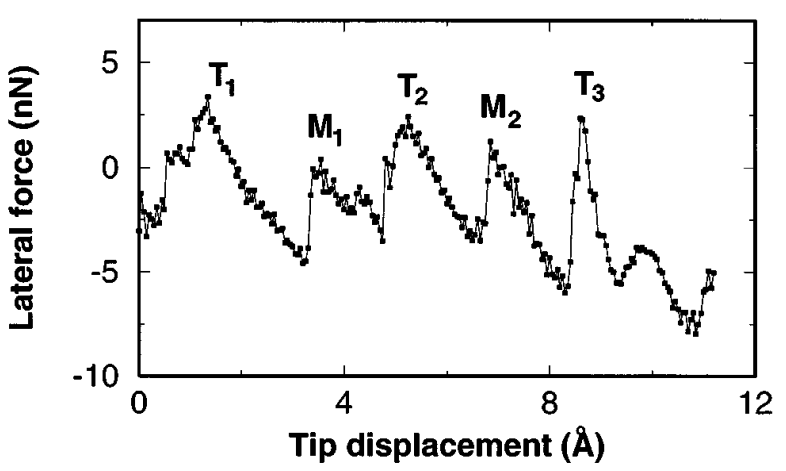

FIG. 1. Variation of the lateral force $F_{L}$ with the displacement $x$ of the asperity. The zero of displacement is taken as the position of the steady-state contact before the start of the sliding. The different transitions (features) in each period is indicated by $T$ and $M$.

atom at the apex and the subsequent layer disappears leaving only eight Ni layers including the contact layer at the surface of the substrate. While some $\mathrm{Ni}$ atoms substitute $\mathrm{Cu}$ atoms at the contact, some of them are accumulated behind the asperity. On the other hand, some $\mathrm{Cu}$ atoms migrate into the $\mathrm{Ni}$ asperity, perhaps due to the fact that their binding energy in the asperity is larger than that on the surface. The $\mathrm{Cu}(110)$ surface at the contact region can be viewed as a $\mathrm{Cu}-\mathrm{Ni}$ alloy with the lattice structure of the $\mathrm{Cu}(110)$ surface. The cohesive energy, melting temperature and the bulk modulus of $\mathrm{Ni}$ is $\sim 30 \%$ higher than those of $\mathrm{Cu}$. In spite of that, two layers of the Ni pyramid which are adjacent to the $\mathrm{Cu}(110)$ surface have changed from the $\mathrm{Ni}(111)$ lattice structure and have become matched to the $\mathrm{Cu}(110)$ substrate. Interestingly, even the distance between these layers comply with that of the $\mathrm{Cu}(110)$ planes. This is perhaps due to the lower coordination numbers of the $\mathrm{Ni}$ atoms near the apex. The remaining top five layers of $\mathrm{Ni}$ asperity keep their original $\mathrm{Ni}(111)$ lattice structure. The effect of the nanoindentation is found in a conical region of the substrate under the asperity, which undergoes a compressive strain.

The sliding started only after the atomic configuration of the contact reached the steady-state condition. The variation of the lateral force $F_{L}$ with the displacement is shown in Fig. 1. Here, the sign of $F_{L}$ specifies only its direction, and by definition, $F_{L}<0$ is in the opposite direction to the motion of the asperity. Striking features that one observes from this force variation are as follows: (i) The abrupt changes of the $F_{L}(x)$ curve display a quasiperiodic variation with a period of approximately $3.5 \AA$. Two features of different characters (labeled $T$ and $M$ ) occur consecutively in each period; these are noticed by the fact that $\left|F_{L}(x)\right|$ decreases abruptly with the displacement of the asperity; even it changes sign momentarily. (ii) Between two consecutive features (from $T_{n}$ to $M_{n}$ or from $M_{n}$ to $\left.T_{n+1}\right)\left|F_{L}(x)\right|$ increases with displacement $x$; it displays a quasielastic behavior. (iii) The maximum value of $\left|F_{L}(x)\right|$ at the end of each quasielastic region increases with increasing number of period $n$. The work done against $F_{L}(x)$ over a period from $T_{n}$ to $T_{n+1}$, i.e., $-\int_{T_{n}}^{T_{n+1}} F_{L}(x) d x$, increases from period to period. This deviation from the Amonton's law is explained by the growth of the junction. ${ }^{12}$ The variation of lateral force showing the elastic (or linear) region followed by an abrupt decrease is a peculiarity of the stick-slip process in dry sliding friction that occurred in certain ideal conditions so far. Clearly, the variation of $F_{L}(x)$ in Fig. 1 is not as regular as that observed on the graphite surface. ${ }^{7}$ Nonetheless, it is of particular interest since the sliding of a metal asperity on an incommensurate metal surface is shown to give rise to a stick-slip behavior. Since the asperity forms a finite size contact with the ordered structure, the occurance of a quasiperiodic stick-slip behavior cannot be explained by the sharpness of the asperity. It is rather due to the fact that the cubic lattice parameters of $\mathrm{Ni}$ and $\mathrm{Cu}$ vary within $\sim 2.5\left(a_{\mathrm{Ni}}=3.52 \AA\right.$ and $a_{\mathrm{Cu}}=3.61$ $\AA)$. Moreover all edges of the (111) unit cell have the same length as the shorter edge of the (110) unit cell $(a / \sqrt{2})$. Under these circumstances, the sliding of a $\mathrm{Ni}$ asperity follows a special path on the Born-Oppenheimer surface that passes through certain structural phases explained below. However, we expect that the stick-slip behavior in Fig. 1 can be washed out if the sliding involves multiple asperity. Very large contacts (that is unusual for an asperity) can give rise to misfit dislocation.

Side views and $x y$ projection of the atoms in the layers are shown in Fig. 2, only for $T_{1}, M_{1}$, and $T_{3}$ during sliding of the $\mathrm{Ni}$ asperity. Figure 2(a) depicts atomic configuration essentially before $T_{1}$, but after the onset of contact. As a result of sliding, the top four planes moved and were also slightly lowered. This changed the registry between the top fourth and fifth $\mathrm{Ni}(111)$ layers; the energy of the system is raised and perhaps exceeded that of another structure. Eventually, as shown in Fig. 2(b), the fifth layer from the top, which was initially commensurate with the $\mathrm{Ni}(111)$ layers, undergoes a structural change and becomes matched to the lower Ni layer that was already changed to the lattice structure of the $\mathrm{Cu}(110)$ plane. This structural transition started from the back and propagated to the front of the asperity. Lateral motion of atoms in this particular layer which changed its lattice structure at $T_{1}$ is shown in Fig. 2(c). One row of $\mathrm{Ni}$ atoms moved sideways, the next went ahead, and third one merged to the lower layers. During this incommensurate-commensurate structural transition, while part of the stored energy is kept in the new phase, the rest of it is released in the nonadiabatic transition and dissipated mainly by exciting nonequilibrium phonons. The amount of energy dissipated in this transition is calculated $\sim 3 \mathrm{eV}$. The force $F_{L}(x)$, which was initially resisting the sliding, is lowered suddenly during the transition and changed its direction. The variation of $F_{L}(x)$ associated with $T_{1}$ is reminiscent of the stick-slip motion, but the rearrangement of the atoms leading to an ordered structural transition is rather unexpected. After the transition $T_{1},\left|F_{L}(x)\right|$ increases quasilinearly with the displacement of the asperity. Once the asperity moves approximately $2 \AA$, or by the height of the twodimensional (2D) hexagonal unit cell of the $\mathrm{Ni}(111)$ planes, the stick behavior ends and the slip starts with the second structural transition $M_{1}$. The side views of the atoms before and after the transition in Figs. 2(d)-2(e) and their $x y$ projections in Fig. 2(f) show that two asperity layers both commensurate with the $\mathrm{Cu}(110)$ plane [i.e., one produced by the transition $T_{1}$ above and the $\mathrm{Ni}(110)$ layer below it] merge into a single (111) layer of Ni. The transition starts in front of the $\mathrm{Ni}$ asperity and ends at its back, and $F_{L}(x)$ decreases abruptly. Subsequent to merging some atoms are displaced 


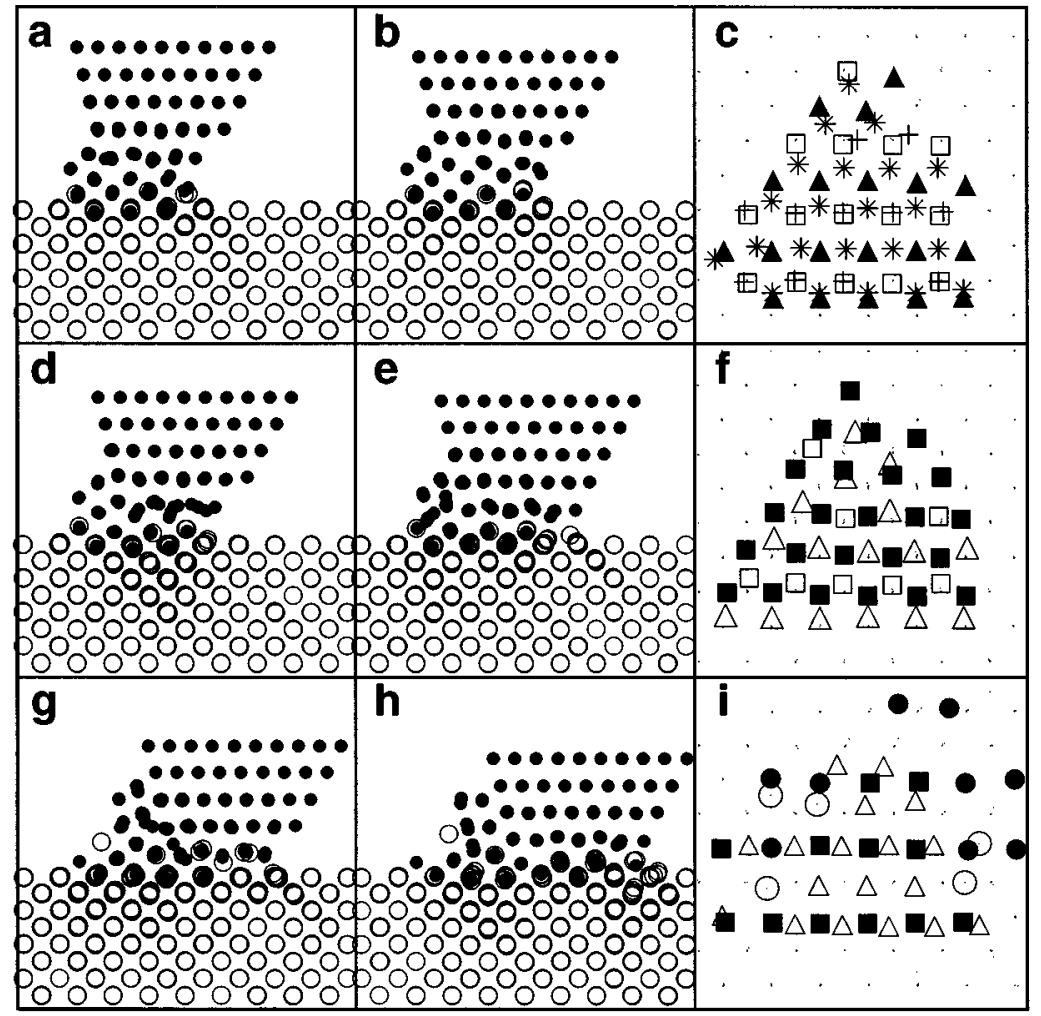

FIG. 2. Side views (panels in the first and second columns) and the $x y$ projections of atoms (panels in the third column) during sliding. (a) and (b) are before and after $T_{1}$; (c) is the corresponding $x y$ projection with the star, plus sign, filled triangle and square denoting the atoms in the fifth and sixth layers of (a) and the fifth and sixth layers of (b) respectively. (d), (e), and (f) are the same for $M_{1}$, with the triangle, square and filled square denoting the $x y$ projections of the atoms in the fifth and sixth layers of (d), and fifth layer of (e), respectively. (g), (h), and (i) are the same for $T_{3}$ with the triangle, circle, filled square, and filled circle denoting the $x y$ projections of the $\mathrm{Ni}$ and the $\mathrm{Cu}$ atoms in the fifth layer of $(\mathrm{g})$ and $(\mathrm{h})$, respectively. The $x$ direction is from left to right in the side views and from bottom to top in the $x y$ projections.

for long distances $(\sim 2 \AA)$ within a short time interval, so that the structural transition leading to a new $\mathrm{Ni}(111)$ plane is eventually completed. We note another interesting feature that upon this latter structural transition, one layer of asperity disappears and hence the slip ends with a wear. During the stick stage from $T_{1}$ until the onset of $M_{1}$ the energy of motion is converted into the potential energy and is stored as the strain energy of $\Delta E_{s} \sim 2.8 \mathrm{eV}$. While part of $\Delta E_{s}$ is kept in the new phase on the Born-Oppenheimer surface as a result of the structural transition labeled by $M_{1}$, the major part, $\Delta E_{d} \sim 2 \mathrm{eV}$, is released to generate local nonequilibrium phonons and hence is dissipated in the whole system. After $M_{1}, F_{L}(x)$ increases again linearly with the displacement until the next period that starts with the transition $T_{2}$. The character of physical phenomena in the second period including $T_{2}$ and $M_{2}$ are essentially the same as the previous one. At each period, the asperity loses one layer, so its contact area with the $\mathrm{Cu}(110)$ surface further increases. The side views of atoms in Figs. 2(g) and 2(h) before and after $T_{3}$ and their $x y$ projections in Fig. 2(i) describe essentially similar structural transformations as explained for $T_{1}$. We terminate the sliding, however, at $M_{3}$ owing to limited extent of asperity and the $\mathrm{Cu}(110)$ surface in our simulations.

The incommensurate-commensurate structural transition shown in Fig. 2 have interesting features. The transition occurs in a short time interval; starting from one ordered 2D structure it ends up in a different ordered atomic structure. In the course of these transitions, the energy of the system makes a sudden change. These features suggest that each structural transition is associated with a first-order phase transition and corresponds to local minimum in the BornOppenheimer surface. This conclusion is strengthened by the analysis of the structure factor $S(\vec{q})$. According to this stan- dard procedure, ${ }^{20}$ the magnitude and sharpness of the $S(\vec{q})$ peaks calculated for a phase consisting of $N$ atoms is taken as a measure of the order parameter. Defining the autocorrelation function $P(\vec{r})=\Sigma_{i, j} \delta\left(\vec{r}+\vec{R}_{j}-\vec{R}_{i}\right)$ in terms of the position vectors $\vec{R}_{i}$ and $\vec{R}_{j}$ of the atoms, the structure factor becomes $S(\vec{q})=N^{-1} \int P(\vec{r}) e^{i \vec{q} \cdot \vec{r}} d \vec{r}$. Figure 3 illustrates the calculated structural factors for the 2D structures examined in Fig. 2. That the atoms form a phase with a well defined lattice structure becomes clear by the peaks of $S(\vec{q})$ which are arranged on the corresponding reciprocal lattice. The phase transition specified by $T_{1}$ is seen in Figs. 3(a) and 3(b) by the transformation from the (111) lattice to the (110) lattice. Through the phase transition $M_{1}$, two ordered (110) lattice structures in Figs. 3(b) and 3(c) are combined and made a new (111) lattice structure that is described in Fig. $3(d)$.

In conclusion, the contact and dry sliding friction described above exhibit several interesting features:

(i) Earlier, the ballistic electron conductance of a contact established between a sharp tip and metal sample have been measured by scanning tunneling microscopy (STM). ${ }^{17}$ The observed variation of conductance in the course of nanoindentation has been related to the discontinuous growth of contact. ${ }^{18}$ In the present study, the junction between the asperity and the $\mathrm{Cu}$ surface is growing with sliding. The cross section of the contact is in the range of $S \sim \pi \lambda_{F}^{2}$, where the electronic states are quantized in the $x y$ plane with a level spacing $\sim 10^{3} \mathrm{~K}$. The number of contact states below $E_{F}$ depends strongly on $S$ and its shape, and the conduction channels derived therefrom are intermixed owing to the tunneling and scattering. ${ }^{19}$ Therefore, the ballistic conductance of the investigation of large and complex systems involving 

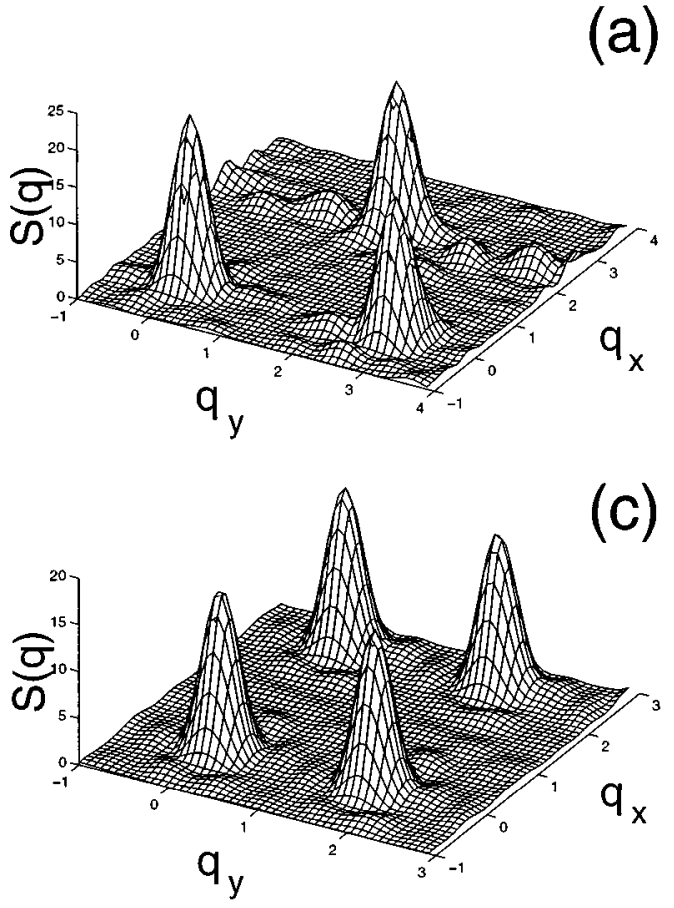

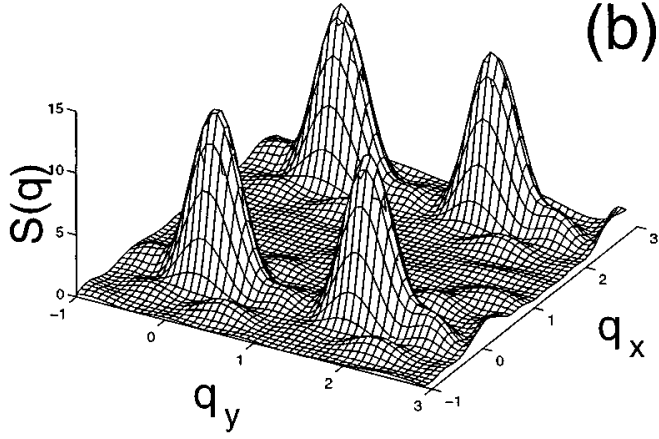

(d)

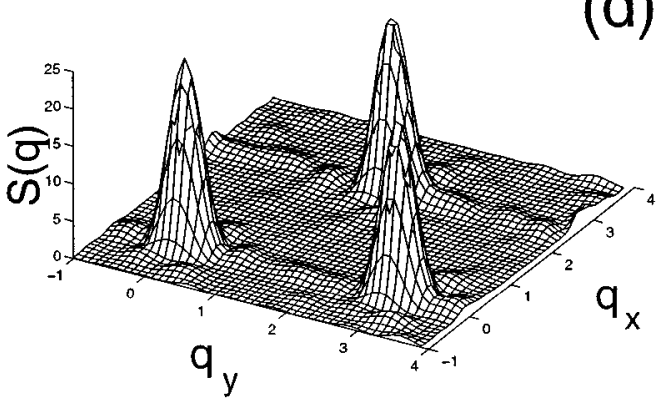

FIG. 3. Structure factors $S(\vec{q})$ calculated for the structural transitions $T_{1}, M_{1}$. The panels a, b, c, and $\mathrm{d}$ are $S(\vec{q})$ versus $\vec{q}$ plots correspond to the specific atomic layers described in the panels a, b, d, and e of Fig. 2, respectively. The unit of $q_{x}$ and $q_{y}$ is $\AA^{-1}$. many contact, $G(x)$ cannot yield sharp quantized step structure even if the contact would evolve adiabatically with sliding. However, $G(x)$ is expected to show abrupt jumps only due to the discontinuous growth of the junction through the $T$ - and $M$-type phase transitions in the course of sliding. By measuring $F_{L}(x)$ and $G(x)$ simultaneously in the course of sliding one can provide additional information about the contact. (ii) As the sliding continues, a cluster of atoms grows at the contact behind the asperity. The atoms of this cluster are not usually in registry with the layers of the asperity, but they change between disordered (or melted) and ordered states in the course of sliding. (iii) It is shown that two different stick-slip motion resulting in $T$ - and $M$ - type structural phase transitions occur within $\sim 3.5 \AA$ translation of the metal asperity on a flat and incommensurate metal surface. Moreover, this process repeats quasiperiodically each time resulting in the wear of one layer of asperity. ${ }^{21}$ The damping of energy is normally reduced because of those incommensurate-commensurate, first-order phase transitions; the structural transitions at the interface play the role of a boundary lubrication.

We thank Dr. I. P. Batra and Professor C. Yalabik for their helpful discussions. A.B. thanks IBM Corporation for the time provided in parallel computer facility at IBM-SP Brifing Center at Almaden Research Laboratory in San Jose, California.
${ }^{1}$ F. P. Bowden and D. Tabor, Friction and Lubrication (Methuen, London, 1965); E. Rabinowicz, Friction and Wear (Wiley, New York, 1965).

${ }^{2}$ M. Schoen et al., Science 245, 1223 (1989); J. Krim et al., Phys. Rev. Lett. 66, 181 (1991); P. A. Thompson et al., ibid. 68, 3448 (1992)

${ }^{3}$ F. F. Abraham et al., Phys. Rev. Lett. 60, 1314 (1988); U. Landman et al., Surf. Sci. 210, L177 (1989); U. Landman, et al., Science 248, 454 (1990).

${ }^{4}$ A. P. Sutton and J. B. Pethica, J. Phys. Condens. Matter 2, 5317 (1990); J. A. Nieminen et al., Acta. Metall. Matter. 40, 2503 (1992).

${ }^{5}$ M. Cieplak et al., Science 265, 1209 (1994).

${ }^{6}$ G. Binnig et al., Phys. Rev. Lett. 56, 930 (1986).

${ }^{7}$ C. M. Mate et al., Phys. Rev. Lett. 59, 1942 (1987).

${ }^{8}$ B. N. J. Persson and A. I. Volokitin, Chem. Phys. Lett. 185, 292 (1991); in Physics of Sliding Friction, edited by B. N. J. Persson and E. Tosatti (Kluwer Academic, Dordrecht, 1996), Vol. E311, p. 253; J. B. Sokoloff, ibid. p. 217; C. Daly and J. Krim, Phys. Rev. Lett. 76, 803 (1996).

${ }^{9}$ D. Tomanek et al., Europhys. Lett. 15, 887 (1991); S. Ciraci et al., Phys. Rev. B 46, 10411 (1992).

${ }^{10}$ H. Matsukawa and H. Fukuyama, Phys. Rev. B 49, 17286 (1994).

${ }^{11}$ M. R. Sørensen et al., Phys. Rev. B 53, 2101 (1996); M. R. Sørensen et al., Phys. Rev. Lett. 77, 5067 (1996).

${ }^{12}$ S. R. Cohen et al., J. Vac. Sci. Technol. A 8, 3449 (1990)

${ }^{13}$ D. Tabor, Proc. R. Soc. London Sect. A 251, 378 (1959).

${ }^{14}$ A. Buldum and S. Ciraci, Phys. Rev. B. 55, 2606 (1997).
${ }^{15}$ M. S. Daw and M. I. Baskes, Phys. Rev. B 29, 6443 (1984); S. M. Foiles et al., ibid. 33, 7983 (1986); M. S. Daw, ibid. 39, 7441 (1989). We used the embedded atom model potentials for $\mathrm{Cu}$ and $\mathrm{Ni}$ developed in the above works.

${ }^{16}$ This "thermostat" allows the local heating in the junction to some extent. See, for example, A. P. Sutton, Curr. Opinion Solid State Mater. Sci. 1, 827 (1996).

${ }^{17}$ J. K. Gimzewski and R. Möller, Phys. Rev. B 36, 1284 (1987).

${ }^{18}$ S. Ciraci and E. Tekman, Phys. Rev. B 40, 11969 (1989). Later the atomic structure of a contact between the STM tip and metal surface has been simulated and discontinuous growth of the contact has been demonstrated. See, T. N. Todorov and A. P. Sutton, Phys. Rev. Lett. 70, 2138 (1993).

${ }^{19}$ E. Tekman and S. Ciraci, Phys. Rev. B 43, 7145 (1991); H. Mehrez et al., ibid. 55, R1981 (1997).

${ }^{20}$ N. E. Cusack, The Physics of Structurally Disordered Matter (Hilger, Bristol, 1987).

${ }^{21}$ The possibility of phase transition of noble gas atoms between two metal surfaces in relative motion has been shown earlier. See, for example, $\mathrm{P}$. A. Thompson and M. O. Robbins, Science 250, 792 (1990); B. N. J. Persson, Phys. Rev. B 48, 18140 (1993); M. Schoen, Computer Simulation of Condensed Phases in Complex Geometries (Springer-Verlag, Berlin, 1993). As noted the situation in the present study is, however, different. 